\title{
Deploying Semantic Web Technologies for Work Integrated Learning in Industry - A Comparison: SME vs. Large Sized Company
}

\author{
Conny Christl ${ }^{1}$, Chiara Ghidini ${ }^{2}$ Joanna Guss $^{3}$, Stefanie Lindstaedt ${ }^{4}$, \\ Viktoria Pammer ${ }^{4}$, Marco Rospocher ${ }^{2}$, Peter Scheir ${ }^{4}$, and Luciano Serafini ${ }^{2}$ \\ ${ }^{1}$ ISN-Innovation Service Network, Zg. Hajdina 159, 2250 Ptuj, Slovenia \\ conny.christl@innovation. at \\ 2 FBK-irst. Via Sommarive, 18. 38100 Trento, Italy \\ \{ghidini,rospocher, serafini\}@fbk.eu \\ ${ }^{3}$ EADS France - Innovation Works. 12, Rue Pasteur - BP76 - 92150 Suresnes, France \\ joanna.guss@eads.net \\ ${ }^{4}$ Know-Center and Graz University of Technology, Knowledge Management Institute \\ Inffeldgasse 21a, 8010 Graz, Austria \\ \{slind, vpammer, pscheir\}@know-center . at
}

\begin{abstract}
Modern businesses operate in a rapidly changing environment. Continuous learning is an essential ingredient in order to stay competitive in such environments. The APOSDLE system utilizes semantic web technologies to create a generic system for supporting knowledge workers in different domains to learnwork. Since APOSDLE relies on three interconnected semantic models to achieve this goal, the question on how to efficiently create high-quality semantic models has become one of the major research challenges. On the basis of two concrete examplesnamely deployment of such a learning system at EADS, a large corporation, and deployment at ISN, a network of SMEs-we report in detail the issues a company has to face, when it wants to deploy a modern learning environment relying on semantic web technology.
\end{abstract}

\section{Introduction}

In the past years we observed a slow but steady uptake of semantic web technologies in businesses. Increasingly search capabilities, data integration and web service communication enabled by semantic web technologies lead to improved business processes, savings in cost and time and heightened efficiency and competitiveness. However, obtaining the needed semantic models has remained a challenge and an art. This challenge is aggravated in situations where not only one model e.g. an ontology, is needed, but a whole network of models needs to be created and later maintained. This contribution reports about the experiences gained during the creation of three interlinked models (process, domain and learning goal model) at two application organizations (EADS and ISN). 
These experiences were obtained in the context of the EU-funded integrated project APOSDLE1 (Advanced Process-Oriented Self-Directed Learning Environment). APOSDLE aims at improving the productivity of knowledge workers by providing learning support during work task execution within the everyday work environment of the user, and by utilizing general organizational resources (documents as well as people) for collaborative learning.

This new learning paradigm of technology-enhanced work-integrated or organizational learning (see [1] for possible scenarios) puts one requirement on technology in the center of attention: Flexibility. In contrast to traditional elearning systems, it is therefore not desirable to create a system specifically matched to one enterprise and one domain. The developed software system must be as generic as possible. Deployment of the system in a new organization or in a new domain must not require substantial software changes.

Further analysis shows that in a system envisioned as APOSDLE, domainspecific knowledge must exist in some form. The system must know about different users, their competencies and learning needs and about the tasks users perform and which digital resources (text documents, multimedia documents etc.) are helpful in which situation (user/task/learning need).

Within the project APOSDLE environments are employed at four application partners. We focus on two of them that also represent two extremes, EADS a large enterprise and ISN a network of SMEs. Although we describe the experiences related to a specific system, we think that our reports are of interest to a wider audience, as APOSDLE relies on many "standard" semantic web technologies and consequently inherits both their advantages and disadvantages.

\section{APOSDLE Semantic Web Technologies}

Semantic web technologies enable switching the learning domain of APOSDLE without software changes, and without hand-crafting domain-specific learning material.

Instead, customization to a domain of application happens by the creation of formal models that capture the necessary aspects of the domain of application. These formal models encapsulate the knowledge that otherwise would be implemented within the program code. In APOSDLE Changing the domain of application means just changing the models.

In general, different formalisms for encoding domain-specific knowledge could have been used, that would not count as "semantic web technologies" as understood by the semantic web community. However, at the time of project start of APOSDLE, description logics and OWL seemed the most broadly developed and advanced technology, with a lot of supportive tools such as ontology editors, reasoners, programming frameworks and APIs, and well-understood theory. Learning material is created ad-hoc by analyzing available organizational resources (textmining) and reusing it.

\footnotetext{
${ }^{1}$ APOSDLE-Advanced Process-Oriented Self-Directed Learning Environment,
} http://www.aposdle.org 


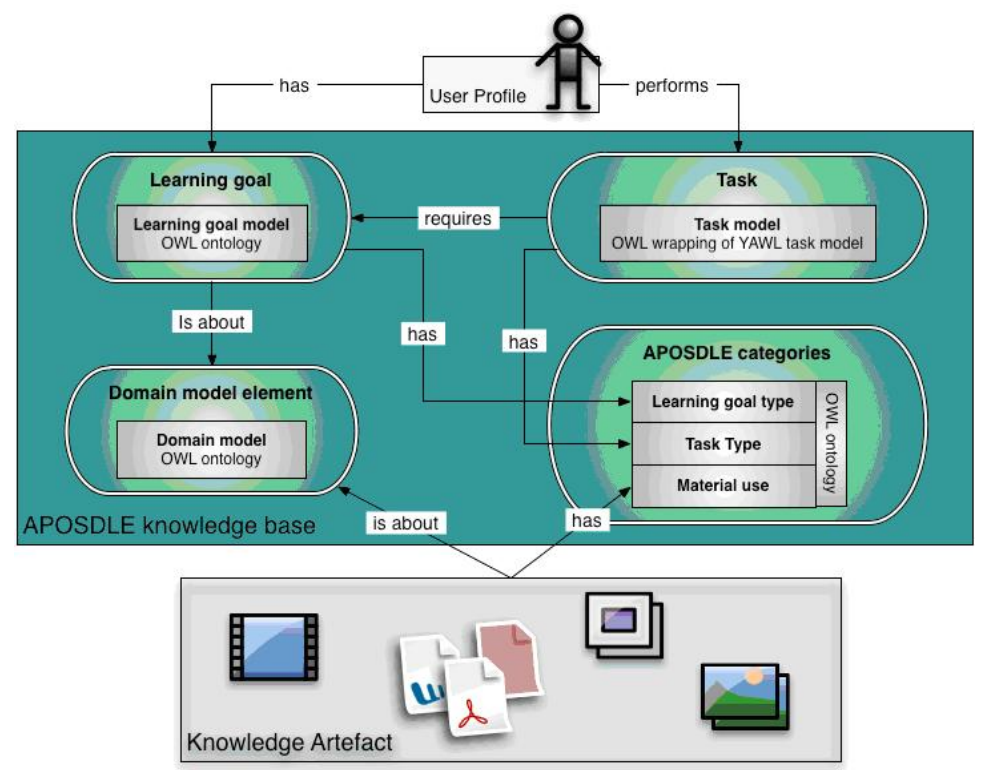

Fig. 1. The APOSDLE Knowledge Base and its relation to other components

In the following we illustrate the realization of this hybrid approach within the APOSDLE system: Firstly, we illustrate the (integrated) schema of semantic models developed inside APOSDLE and proposed as a basis for categorization and retrieval in work-integrated learning, then we illustrate the technology and techniques used to support the construction of these semantic models, and thirdly we illustrate the approach used to connect the semantic models to the resources of the organizational memory (classification).

\subsection{The Semantic Models}

One of the key problems to solve when trying to build a flexible generic system is to identify the basic knowledge that the system must have in order to deliver the worker with context-sensitive learning events, tailored to her specific learning goals, work situation and learning needs. The model we propose, hereafter called APOSDLE knowledge base, is depicted in Fig. 1. In a nutshell, the APOSDLE Knowledge Base contains all the necessary information about the tasks a user can perform in a certain organization, the learning goals required to perform certain tasks, a description of the domain of affairs (application domain) of the organization, and, finally, specific APOSDLE categories used to classify tasks, learning goals and learning resources. The main idea is that starting from the context-sensitive situation of a user,

which includes her current task(s), APOSDLE is able to determine the learning goals of the user and then use the information contained in the APOSDLE 
Knowledge Base to select appropriate resources, so called knowledge artifacts and transform them into learning event:2 proposed to the user for attaining the missing learning goals [2].

A knowledge artifact (KA) is defined in APOSDLE as a document, or part thereof, together with two types of metadata: the learning domain concept addressed / described within the document (piece) and the material use typ 3 of the document (piece) [2].

\subsection{Building the Semantic Models}

Only one of the four models depicted in Fig. 1, the APOSDLE Categories, is an APOSDLE-inherent structure. The others are by nature domain / organization dependent and need to be provided every time the APOSDLE system is configured and deployed for a new organization / domain.

Despite the recent advances of formal modeling and semantic web, we can safely assume-and this is the situation we had to face within the running of the project-that most of the organizations interested in using the APOSDLE system neither have formal models already available, nor all the skills needed to develop them. Therefore, as part of the APOSDLE project we have developed an Integrated Modeling Methodology (IMM)[3] as a series of steps, techniques and tools to support the construction of the semantic models depicted in Fig.1. To be effective and tailored to the APOSDLE system, such a methodology had to satisfy some important requirements:

Provide the organization with high level tools useful to specify knowledge in natural language, to minimize the need to become familiar with formal languages. Support an integrated development of the three models (domain, tasks, and learning goals) in order to ensure a conceptual consistency among these models and an easy formal integration. Support the creation of formal models which are described using different languages (YAWL and OWL)Encourage knowledge engineers and domain experts from the organization to work together in a collaborative manner and become the main actors of the modeling phase 4 .

The first important semantic technology used to support modeling inside APOSDLE is a semantic wik 5 . The choice of a semantic wiki as the main tool for informal modeling was made for several reasons: first it provides a state of the art collaborative tool which has made possible an active collaboration among all the actors involved in the modeling activities; second it provides a uniform tool for the informal specification of the different models (domain model, task model, learning goal model) using natural language; third, the natural language

${ }^{2}$ Learning events are a kind of amalgamation of knowledge artifacts following instructional principles. To read more about learning events, see for instance [2].

${ }^{3}$ Within APOSDLE five material use types have been defined: The material use "Definition", "Example", "Howtodo", "Main points", "More about" [2].

${ }^{4}$ In the event of unavailable knowledge engineers, or knowledge engineers not enough skilled to follow the process in an autonomous manner, we provided some coaching.

${ }^{5}$ We used Semantic MediaWiki (http://www.semantic-mediawiki.org). 
descriptions inserted in a semantic wiki can be structured according to predefined templates, and with the help of semantic constructs like attributes and relations. As a consequence the informal descriptions in natural language contain enough structure to be automatically, or semi-automatically, translated in OWL ontologies, thus allowing the reuse of informal descriptions for automatic ontology creation.

The second technology used to support informal modeling (and in particular knowledge elicitation) within APOSDLE were techniques of term extraction to provide lists of candidate concepts. These techniques, illustrated in [4] [2] have also proven useful to reduce the burden of modeling and speed up the process.

The informal models described in the semantic wiki were translated to formal models.

- The domain model was (semi-) automatically translated to an OWL ontology,

- The task model was formalized by using YAWL [5]. This had to be done manually,

- The learning goal model was formally specified with a custom tool, the TaskCompetency Tool (TACT for short $\sqrt[6]{6}$ by connecting relevant learning goal 47 to tasks.

\subsection{Connecting Semantic Models and Resources}

To allow for retrieving learning content the formal models have to be connected to the resources of the respective organization (annotation). The annotation process is one of the key challenges in any system that relies on semantic annotations. Semantic annotations in the present system are based on the Aboutness of resources [6]. This means that we annotate (parts of) documents with a set of concepts the content of the document is about. Basically, two options exist.

- Manual annotation: The resulting annotations are probably of good quality, given that motivated and competent persons perform the annotation. The large drawback is the large effort that manual annotation requires.

- Automatic annotation: The resulting annotations are of lower quality than manual annotation given any state-of-the art technology of natural language processing or statistically-based classification. The advantage is of course that it is faster.

In APOSDLE, manual annotation is used to create an initial set of knowledge artifacts of good quality. These are used on the one hand as high-quality learning

${ }^{6}$ This Tool is developed by APOSDLE Team [2].

${ }^{7}$ A learning goal is regarded as the combination of a "Learning goal Type" and a "Domain Concept". Learning goal types specify the type and degree of knowledge and skills the person must or typically wishes acquire about this topic. Within APOSDLE Learning Goal Types are "Remember", "Understand", "Create" and "Apply". The domain concept defines the content or topic that the learning goal is about. 
material, and on the other hand as training material for automatic classification. Manual annotation can be performed again during usage of APOSDLE, when a user opens a retrieved knowledge artifact and wants to edit it. Automatic annotation is performed at regular intervals. Assignment of domain concepts can be seen as classical text classification problem of assigning documents to a set of predefined classes [7]. A basic algorithm, using Support Vector Machines (SVM) and k-Nearest neighbor algorithms, has been modified to incorporate knowledge about hierarchical relations between the classes, i.e. the domain concepts assigned to pieces of text [8].

Retrieval of learning content can be performed based on the semantic annotations. A detailed description of the approach taken in APOSDLE for retrieval and further references are described by Scheir, Ghidini and Lindstaedt [9].

\section{APOSDLE in Application Context}

APOSDLE is adapted to four application partner domains. In this section the two most oppositional are compared to each other. On the one site EADS as large company, on the other side ISN, as network of SMEs. The main focus of our attention in this section is on describing shortly the application domains organization, their motivation for using APOSDLE as well as some major activities necessary to deploy APOSDLE.

\subsection{Application Domains, Challenges and Motivation for Using APOSDLE}

$\mathrm{EADS}^{8}$ is the largest aerospace company in Europe active in the fields of civil and military aircraft, space, defense systems and services. EADS Innovation Works (IW) decided to implement the APOSDLE system in the Simulation Domain. This is due to the growing importance of simulations and the necessity to have quicker operational performances of engineers in this domain. The simulation teams are in charge of designing, developing and achieving numerical simulations of electromagnetic problems or phenomena (Electromagnetic (EM) compatibility, simulations of EM attacks on aeronautical systems or subsystems, etc.). They are composed of engineers who are EM physicists or mathematicians and Soft/Hardware Specialists. The simulation experts are located in different countries and cities and rarely available to help newcomers in learning. Moreover the time allowed to maintain up-to-date worker skills or to acquire new knowledge has to be reduced. The simulation activities require a high level of knowledge and expertise. EADS IW is emphatically interested to introduce an innovative task- and process-oriented learning tool such as APOSDLE to support the simulation development process.

On the other side ISN-Innovation Service Networl 19 is a service and research company in the field of innovation- and knowledge management composed of a

\footnotetext{
${ }^{8}$ EADS-European Aeronautic Defense and Space Company EADS N.V., http:// www.eads.net

${ }^{9}$ ISN-Innovation Service Network (ISN), http://www.isn.eu
} 
network of SMEs in Slovenia and Austria. ISN is supplemented by more than 40 further partners from universities, competence centers and service companies acting as a pool of experts. All experts are or intend to be specialized in a few, very specific fields such as specific management methods, patent management, creativity methods, etc. In order to stay competitive at ISN each network partner needs to continuously improve her skills in the focus field, rapidly learn aspects of other fields relevant to one customer project, and apply both within the customer's situation. Since the network partners are increasingly involved in partner projects, the time for learning and improvement of competencies becomes more limited. On the other hand, more in-depth and more diverse know-how is asked for by the customers. Thus, within ISN the focus of the APOSDLE domain is on project processing and management in order to provide specialized knowhow in the area of innovation and knowledge management to their customer organizations.

Currently at both organizations there is no common learning resources database or system that can support the knowledge worker. Learning during work task execution is done by using templates, guidelines, project documentation or internet resources and with few sharing effort to the collective. The initial motivation for implementing APOSDLE at ISN and EADS is therefore to create a common knowledge base which integrates resources from different repositories and backend systems for all knowledge workers in order to improve knowledge acquisition, reuse and sharing.

\subsection{Using Semantic Technology-Deriving from an Analysis of Competitive Approaches}

At the moment there are several different approaches in the field of knowledge management and e-learning which aim at meeting above mentioned challenges.

Desktop search engines (e.g. Google Desktop) or ontology based search engines are often used for knowledge retrieval within organizations (e.g. KINOA 10 at EADS IW). On the other side knowledge sharing is supported by providing collaboration tools like e-mail, document sharing (e.g. Groove is used at ISN) or instant messaging (e.g. Skype is used at ISN). Even social networking platforms support collaborating and sharing knowledge with each other.

Frequently companies have implemented e-learning systems or learn management systems (LMS) in order to provide employees the possibility to further improve their skills. However knowledge worker in very knowledge intensive domains like at EADS or ISN often do not have the time for explicit learning. They need flexible real-time support within their current work task. Additionally developing an e-learning System is an expensive and time consuming task [1].

By using the potential of semantic web technologies successful approaches of knowledge management and e-learning are combined within APOSDLE. Therefore the essential benefit for ISN and EADS by using APOSDLE is:

$\overline{10}$ KINOA-management and sharing of written information content with shared ontologies. 
- Learning during execution of work tasks.

- Collaboratively sharing knowledge within the work environment.

- Learn from resources already available within the organization.

\section{Customization and Usage of APOSDLE}

Both ISN and EADS defined one knowledge engineer (KE), who is responsible for the elicitation of knowledge from the domain experts (DE) and guides the entire modeling process. The DE provides the fundamental knowledge about the domain of the users of APOSDLE and their learning needs. The DE also specifies the pool of resources to be used for knowledge extraction.

Modeling corresponds to customizing the existing APOSDLE system to a specific domain, with a focus on exactly those tasks and competencies that are interesting for the users. Modeling is also very often the "bottleneck" in many semantic web applications, i.e. the applications would be good, or would work if only there were enough / more appropriate models. Therefore we think it of interest to describe the modeling that was necessary at ISN and EADS in order to deploy and use APOSDLE. We followed the Integrated Modeling Methodology (IMM) [3]. Below we describe the steps prescribed by the IMM and the corresponding experiences at EADS and ISN.

\subsection{Informal Modeling}

Informal modeling follows a knowledge elicitation phase, in which a number of relevant "elements", be it terms, phrases etc. elicited from DE are collected. The goal of the informal modeling phase is that the knowledge engineer enters, after reviewing and filtering them, these first results into a semantic wiki. In a second stage the informal models, i.e. described in natural language, are formalized and connected to each other.

Knowledge elicitation from domain experts. The first stage in APOSDLE models design for Electromagnetic Simulation domain consisted in collecting relevant resources and interviewing experts: At EADS three simulation domain experts (EM physics specialists and mathematics expert) were interviewed separately, during two hours. The discussions focused on simulation process, knowledge needs and difficulties that occur at each simulation development task. As at ISN each domain expert is expert in a very special knowledge field, seven domain experts were interviewed according to a developed guideline asking about tasks they perform, learning needs they have and resources they use for both working and learning. Further the Card Sorting Technique [10] was used to approve the elicited domain knowledge.

Knowledge elicitation from resources. As one way of externalizing domain knowledge is in the form of documents written in natural language, documentbased ontology engineering aims at using information available in documents 


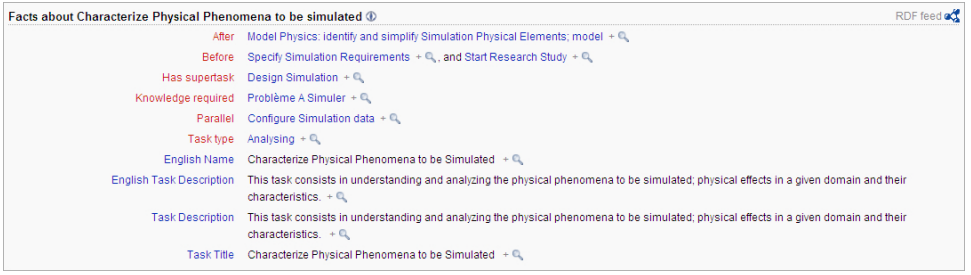

Fig. 2. Filled template for EADS task

to create formal knowledge representations. We used the discovery tab, a plugin for Protg OWL, [4] to discover knowledge from text-documents. Grouping documents to form clusters of different topics, gives an overview of the domain covered by the documents. By using statistical and natural language processing methods, relevant terms are extracted from a set of documents. Furthermore, the terms can be grouped by synonymity. Within discovery tab, standard text mining methods have been used. For relevant term extraction, various pre-processing techniques, such as stop-word lists and stemming are employed [4].

Informal modeling with semantic wiki. Using pre-defined templates we have automatically created a page for each one of the elements (concepts or tasks) of the models we wanted to obtain via the wiki. Thus, the domain experts, knowledge engineers and APOSDLE technology partners located in different cities and countries had the possibility to access the models, refine them or comment. The filled template for a task is shown in Fig. 2 and is tailored to the information we needed to obtain for APOSDLE.

As shown the information required in the template is very basic. Nevertheless it allows guiding the modeling team to provide all the information that was needed by the particular application. In addition templates contained also hints to guide the early alignment of models. For instance the template for task description asks also for knowledge required to perform this task, and this has a clear connection with the domain and learning goal models.

Advantages and Difficulties. The experts were in general very busy and tied up in heavy workloads. However their involvement in modeling activity is strongly required. The knowledge elicitation only from documents risks to build up very theoretical and over-complicated knowledge schemes that passes by real knowledge needs. The main difficulty that occurred at this stage of modeling activity consisted in putting the right definition behind the terms used by experts or extracted from documents. Several terms are common to some experts but used to evoke different concepts: e.g. at EADS the term "model" can mean mathematical model, simulation model, data model, numerical model, etc. Some specialists use also different terms to designate the same concepts. Another problem was a lack of documentation, for instance at EADS a large number of simulation knowledge is not really formalized and can be stored in individul's repositories or on desktops. 
Currently the Discovery Tab [4] is based on WordNet11 and Apache's Wordnet12 package. Thus, it was difficult to automatically extract terms from German (ISN) or French (EADS) documents.

The results of the interviews as well as documents analysis allowed the EADS and ISN Knowledge Engineer to create a very first version of the informal models in the Semantic MediaWiki, define some learning scenarios and knowledge / learning needs. Because of both the complexity the domain has and the information needed for the semantic wiki, the informal modeling activity was quite time and resources consuming. However, it turned out that a semantic wiki is very useful for the internal model revision as it allows queries about special relations, shows up all relations a concept/task has to another concepts/task etc.

For the EADS Simulation domain the informal modeling process required about 2.5 person months. Altogether 43 EADS domain concepts and 22 tasks were identified, classified, and described. The modeling process at ISN required 3 person months and 140 concepts and 31 tasks were defined for describing their domain.

\subsection{Formal Modeling and Resources Annotation}

Based on the KE's semantic descriptions of tasks and concepts in the wiki templates, Semantic MediaWiki generates machine-readable documents in RDF format. These are transformed into an OWL model. The formal domain model could then be viewed and revised by knowledge engineers in the Protégé editor. Basically most of the informally given information was correctly formalizedchecking the formal models some specific relation types (e.g. the relationtype has result) that could not be translated to OWL had to be defined anew. Additionally some concepts arising from not deleted wiki pages in the ontology had to be deleted. The task model was manually defined by technology partners in YAWL [5] starting from the informal descriptions provided in the semantic wiki. The final step for the task model is the application of formal checks provided by the YAWL editor. These checks result in a debugging of the YAWL specification. This outcome is complemented by a transformation of the YAWL specification to an OWL file containing the complete list of tasks [3].

Once the task and the domain model were formalized knowledge engineers linked with the TACT Tool [2] respective concepts and learning goals to every task. For instance for to the task "Characterize Physical Phenomena to be simulated" (Fig.2) the learning goal "Understand" and the concept "Radiation" was defined (among others).

Finally, the resources annotation was carried out using the annotation tool [2](Fig. 2 shows an example. The annotation tool consists of a pdf-viewer plus the custom functionality to create knowledge artifacts (KA), i.e. to assign domain concepts and material use concepts. The annotation process at EADS and ISN

11 http://wordnet.princeton.edu/

12 http://lucene.apache.org/java/2_0_0/api/org/apache/lucene/wordnet 


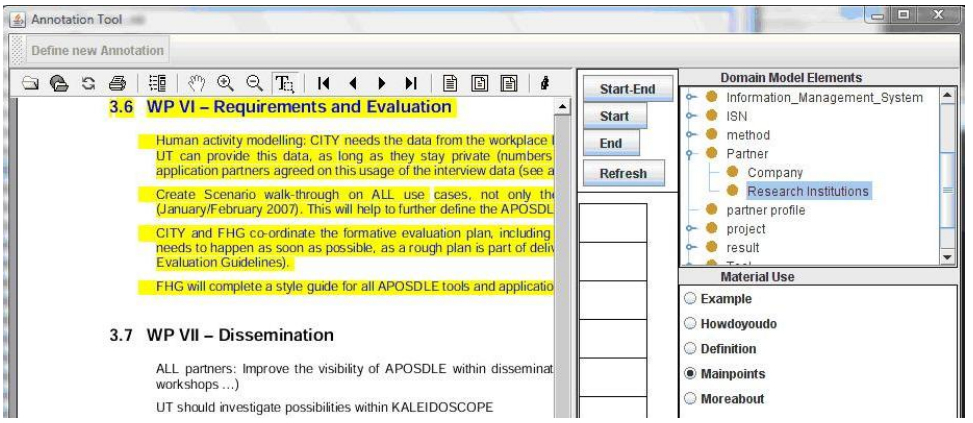

Fig. 3. The Annotation Tool

was composed of 2 main stages. The first one consisted in selecting an initial set of relevant documents (the training corpus). This set of representative resources dealing with the model concepts was manually annotated using APOSDLE annotation tool. For example, at ISN a piece of text within an article containing a definition about the concept "Brainstorming" was annotated with the respective concept and the material use type "Definition". At EADS 60 knowledge artifacts were created from 25 documents. The Knowledge Engineer dedicated 3 days for training corpus building. The results from ISN are very similar. About 100 knowledge artifacts from 50 documents were created within 5 days.

In the second stage the resources annotation process was done automatically. This means that a set of concepts for a document based on a training set of previously annotated documents is suggested. Finally the KE checked these automatic annotations randomly.

Advantages and Difficulties. In total at EADS 150 knowledge artifacts were created from 55 documents. Because of the unavailability of domain experts, the complexity of Simulation Activity and difficulty to collect the right resources the annotation process for APOSDLE Prototype at EADS required about 1.5 person months. At ISN the Annotation process required 1 person moth. And about 200 (KA) were created of 150 documents.

\subsection{APOSDLE in Use}

Both at EADS and at ISN the prototype of the APOSDLE system was not yet connected to other live systems. Especially at EADS are high security and privacy settings, therefore we decided to first test the system itself, before connecting it to other sources. At EADS the APOSDLE Prototype was used by 10 evaluation users located in Toulouse and Suresnes. They had different levels of knowledge of Simulation Domain and different learning objectives. At ISN installation of APOSDLE Prototype was carried out at the Headquarters in Graz and 10 users were integrated in the evaluation process.

As APOSDLE is a quite complex system we decided to accompany the installation with user trainings. These Trainings have been carried out by the respective 
knowledge engineers. Generally users are not familiar with the models based and semantic technology approach used in APOSDLE-thus a lot of conceptual questions concerning tasks and related learning goals as well as retrieved resources that depend on task, learning goal and competency arose.

\section{Challenges for Semantic Web Technologies in Industry}

Although the evaluation of the APOSDLE system is still ongoing and validated assumptions concerning the models and the system can not be made, we figured out the main points to be addressed for future development.

\subsection{Models}

A very important point concerns the granularity of the models. For this prototype we tried to find out a balance between not too generic and not too specialized models. In consequence it was quite difficult to find a right formulation of a task in order to meet all demands like comprehensibility, feasibility, not too complex etc. and at the same time matching this with a task type (some tasks may cover more than one task type) and a specific concept (required knowledge). For instance we decided to model the task "Select appropriate tools for method". The concept "method" has about 15 sub concepts. As it would have been too much effort to model 15 different tasks and assign the related learning goals and concepts, we decided to follow this quite generic way.

However, after using the real system we identified a need to enrich the domain model in order to allow learners and experts acquire / exchange knowledge on more specific topics (e.g. at EADS measures, different kinds of simulation models and resolution methods). Currently we are working on enabling the KE to represent more complex structures in the formal models.

\subsection{Modeling Process}

In addition to models, the modeling process also needs to be improved. Firstly we want to integrate more already existing classification like e.g. files structures. Secondly the Discovery Tab could be enriched by new functionalities, e.g. the possibility of associating to the extracted term the snippets of texts and the documents it comes from.

For next model development we will directly use one semantic wiki for each partner-for this prototype we used one semantic wiki for all application domains and encountered some overlapping in concepts and tasks of different domains. The application domains also want to integrate the possibility of a graphical representation of the models and its relations in the semantic wiki.

\subsection{Resources Annotation}

Based on early results of the evaluation we assume that the quality of the annotations has a huge impact on the users' confidence in APOSDLE system. 
The resources provided by APOSDLE have to be relevant to the learner context (domain, task and especially his/her learning objective). Based on our first impressions the manual resource annotations are very good. Assumptions concerning the automatic annotation can not be made at the moment. Therefore it is an open issue, if it would be worth to spend the effort for annotating all documents manually in the case users are not satisfied with automatically provided annotations. The current approach is, that every APOSDLE user can add, edit or delete an annotation to a document-thus the more users give their feedback on the annotations, the better the documents are annotated. During the evaluation phase we started the discussion whether we should follow this approach. Reasons for this are that (a) it is not easy to annotate a document, some people may not do this because it is too complicated. (b) Domain experts mentioned that adding, editing or deleting an annotation is quite subjective, because e.g. domain experts would annotate a document in a different way as others would do this.

\section{Conclusion}

The paper at hand presented a detailed report of experiences and issues a company has to face, when it wants to deploy a modern learning environment relying on semantic web technologies.

Although the described application domains represent two extremes, both could follow without major differences the same modeling approach in order to deploy a complex system like APOSDLE. Thus, it is not primarily important to which company-whether a large enterprise or a network of SMEs-it is deployed, but to have a clear idea of the domain to be modeled and the users that will use the system. Thus, we will evaluate clear criteria and develop a kind of guideline for which domain and for which granularity of models our modeling approach is suitable.

The IMM approach enables also persons not skilled in knowledge engineering, object-oriented modeling or ontology-building to run through our proposed modeling process. The semantic wiki enabled the knowledge engineer to describe the respective domain in natural language-this information can then (semi-) automatically extracted and translated to OWL. With the development of tools, which can be easily applied in industry-even more business problems could benefit from the potential of semantic web technologies. We will continue to apply and improve the collaborative approach to improve and extend the deployment of semantic technologies in industry.

Acknowledgements. APOSDLE is partially funded under the FP6 of the European Commission within the IST work program 2004 (FP6-IST-2004-027023). The Know-Center is funded within the Austrian COMET Program-Competence Centers for Excellent Technologies-under the auspices of the Austrian Ministry of Transport, Innovation and Technology, the Austrian Ministry of Economics and Labor and by the State of Styria. COMET is managed by the Austrian Research Promotion Agency FFG. 


\section{References}

1. Lindstaedt, S., Mayer, H.: A Storyboard of the APOSDLE Vision. In: Nejdl, W., Tochtermann, K. (eds.) EC-TEL 2006. LNCS, vol. 4227, pp. 628-633. Springer, Heidelberg (2006)

2. Leemkuil, H., de Hoog, R., Lindstaedt, S., Ley, T., Scheir, P., Ulbrich, A., Beham, G., Klieber, W., Aehnelt, M., Lokaiczyk, R., Zinnen, A., Doering, M., Goertz, M., Leitner, L.S.H., Kump, B., Pammer, V.: Conceptual Framework \& Architecture Version 1 (2007),

http://www . aposdle.tugraz .at/media/multimedia/files/ conceptual_framework_architecture_version_1

3. Ghidini, C., Rospocher, M., Serafini, L., Kump, B., Pammer, V., Faatz, A., Guss, J.: Integrated Modelling Methodology (IMM) Version 1 (2007), http://www . aposdle.tugraz.at/media/multimedia/files/ integrated_modelling_methodology_version_1

4. Pammer, V., Scheir, P., Lindstaedt, S.: Two Protg plug-ins for supporting document-based ontology engineering and ontological annotation at documentlevel. In: 10th International Protg Conference (2007)

5. Godehardt, E., Doehring, M., Faatz, A., Goertz, M.: Deploying YAWL for Workflows in Workplace-embedded Learning. In: 7th International Conference on Knowledge Management, pp. 281-288 (2007)

6. Handschuh, S.: Semantic Annotation of Resources in the Semantic Web. In: Semantic Web Services: Concepts, Technologies, and Applications, pp. 135-155. Springer New York, Inc., Secaucus (2007)

7. Sebastiani, F.: Machine learning in automated text categorization. ACM Computing Surveys (CSUR) 34(1), 1-47 (2002)

8. Granitzer, M., Auer, P.: Experiments with Hierarchical Text Classification. In: Proceedings of 9th International Conference on Artificial Intelligence. ACTA Press, Benidorm, Spain, IASTED (2005)

9. Scheir, P., Ghidini, C., Lindstaedt, S.: Improving Search on the Semantic Desktop using Associative Retrieval Techniques. In: Proceedings of I-SEMANTICS 2007, pp. 221-228 (2007)

10. Schilb, S.: Card Sorting-Techniken im Überblick. I-Com 4(1), 49-50 (2005) 